\title{
Simulation modeling of centrifugal concentrator as a basis for increasing its output in enriching industrial waste
}

\author{
Andrey Taskin ${ }^{1 *}$, Oleg Danilov ${ }^{1}$, Viktor Shalanin ${ }^{2}$, Leonid Alekseiko ${ }^{1}$ and Oleg Elkin ${ }^{1}$ \\ ${ }^{1}$ Far Eastern Federal University (FEFU), International Center of Mineral Processing Technologies, \\ Vladivostok, Russia \\ ${ }^{2}$ Far Eastern Federal University (FEFU), School of Engineering, Vladivostok, Russia
}

\begin{abstract}
In the research paper a computer model of the operation of a vibrational concentrator used to separate the slurry into individual fractions of a grain size by depositing particles of specified characteristics in the field of centrifugal forces is created and verified using the methods of computational hydrodynamics with the Ansys software complex. Using the model will make it possible to: 1) to study the operation of concentrators of various sizes and designs under different operating conditions, with a change in hydraulic characteristics (speed of treated liquid, speed of rotation of the bowl); 2) in the future study the problems of depositing suspended particles of specified characteristics (density, size) in a centrifugal field and determine the concentration of the sediment in the treatment process. The results of the work will make it possible to increase the efficiency of the enrichment of ash and slag waste due to a more thorough understanding of interaction mechanisms between the centrifugal concentrator and the enriched geomaterial.
\end{abstract}

\section{Introduction}

The main issues in today's mining industry include significant depletion of existing mineral resource base, complex material composition and constantly deteriorating quality of ore. This circumstance requires the use of bulk mining extraction at mining plants, which in turn leads to a decrease in the content of valuable component in the ore mass. The alternative source of valuable components - geomaterials of industrial origin (for instance, waste generated by power industry, mining and processing plants, gold mining plants) have a similar set of issues: complex, volatile chemical composition and low content of commercially valuable components, uneven distribution in the material. As a consequence, in order to obtain a unit of commercial product, one is required to process a large amount of raw materials [1-3].

One of the technical challenges in this case is a method of increasing the output of concentrators intended for processing of raw materials. At the same time, selection of optimal operating conditions for mineral-processing equipment at a laboratory or in actual

*Corresponding author: taskin@yandex.ru 
industrial conditions is not efficient, as it requires considerable time. An effective solution in the selection of operating modes, according to many experts in the field of enrichment, is the use of computer modeling, in particular simulation [4-7].

This type of modeling makes it possible to quickly assess the effectiveness of certain technological processes in the system under study, the effectiveness of implementing various options for its operation. From the point of view of enrichment, ash and slag waste from power utilities is the most complex object of study. In this connection, the development of a numerical method for calculating hydrodynamic processes in the enrichment of industrial geomaterials in a centrifugal concentrator, as in the case of ash and slag waste, is a priority research and practical problem.

\section{Implementation of the concentrator operation model}

The modeling of the object consisted of the following stages: 1) creation of geometric model; 2) creation of computational grid; 3) adjusting the solver; 4) solving the problem; 5) verification of the mathematical model.

\subsection{Creation of geometric model}

A two-dimensional full-scale axisymmetric model of a centrifuge was selected as a geometric model. The dimensions of the computational area of models are made without distortion, on the basis of actually existing apparatuses. The enriched medium is fed into the central part of the bottom of the concentrator bowl and is distributed to the inclined walls of the bowl by the impeller. The water is withdrawn through the upper edge of the concentrator bowl. Geometric models of concentrators No.1 and No.2 are shown in Fig. 1 and 2 .

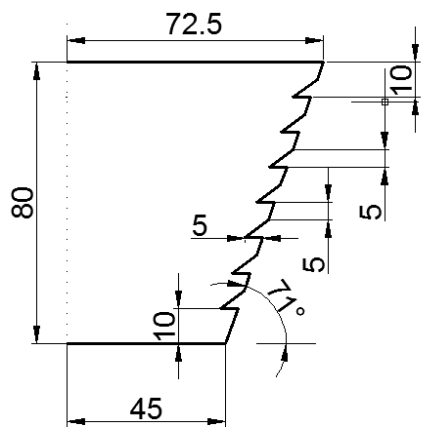

Fig. 1. Geometric characteristics of concentrator model No.1 


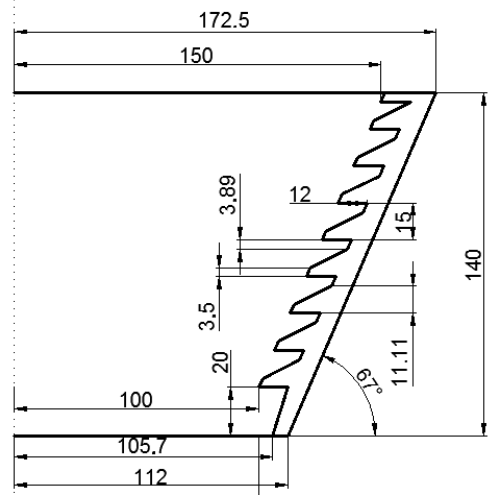

Fig. 2. Geometric characteristics of concentrator model No.2

\subsection{Creation of computational grid}

The computational grid was created using the Delaunay triangulation method, by constructing a primary computational grid on sides with subsequent construction of a computational grid inside the plane of the model. The total number of cells for concentrator No. 1 was 18,900 elements, for concentrator No. 2 - 17500 (Fig. 3, 4).

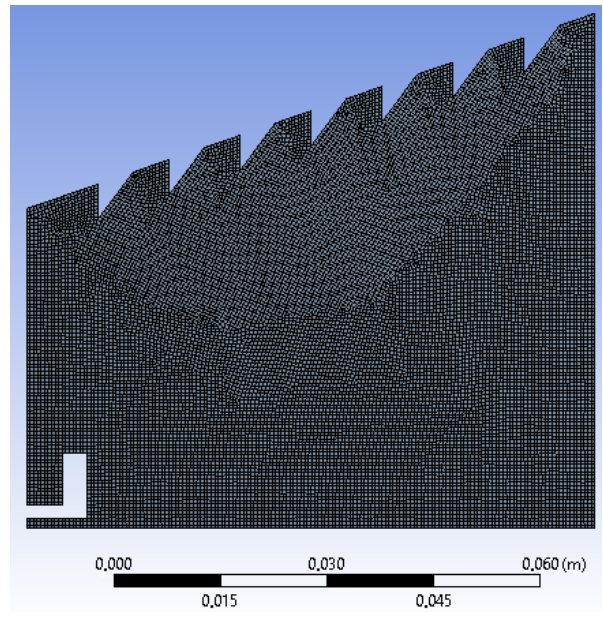

Fig.3. Computational grid for concentrator model No. 1

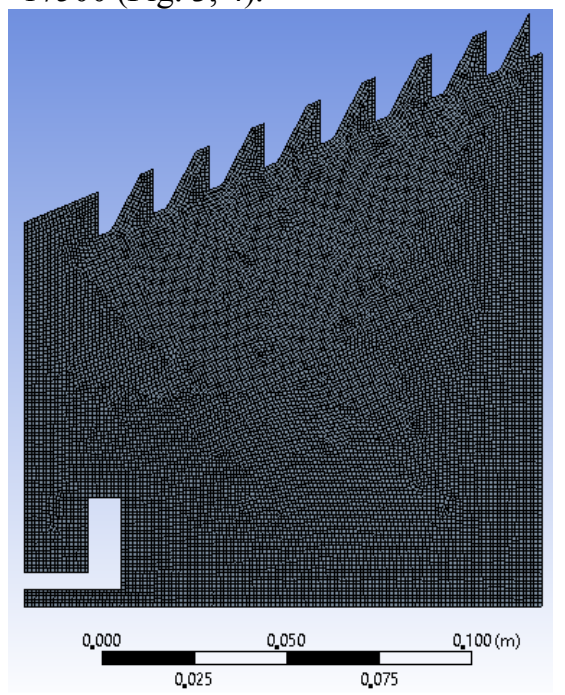

Fig.4. Computational grid for concentrator model No. 2

\subsection{Adjusting the solver}

The problem solving was carried out by means of ANSYS FLUENT software. The problem under consideration is nonstationary.

For the mathematical description of turbulence, the k-omega Standard model was used. A mathematical model VOF (Volume of Fluid) with the number of phases $=2$ and Implicit Formulation was used to model the tangential water-air discontinuity surface.

The flow characteristics at the "inlet" and "outlet," as well as the direction and the number of revolutions of the concentrator bowl were considered as boundary conditions. In 
order to implement the joint solution of the velocity-pressure equations, PISO algorithm was used, which is most suitable for solving non-stationary problems.

\subsection{Solving the problem}

Problem solving was carried out by achieving convergence of the solution at each time interval. The time interval varied from $0.0001 \mathrm{~s}$ to $0.001 \mathrm{~s}$. The increase in the time interval was used to reduce the total solution time. The number of iterations at each step did not exceed 50 .

\section{Results and discussion}

\subsection{Results of problem solving}

As a result of the calculations, liquid flow patterns with a water-air interface and trajectories of flow lines were obtained. The figures present: Fig. 5 - flow pattern of liquid in the concentrator No. 1; Fig. 6 - flow lines in the concentrator No. 1; Fig. 7 - flow pattern in the concentrator No. 2.

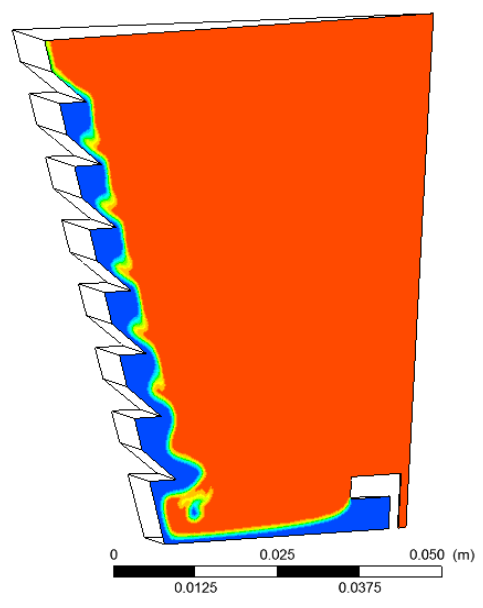

Fig. 5. Flow pattern of liquid in the concentrator No. 1

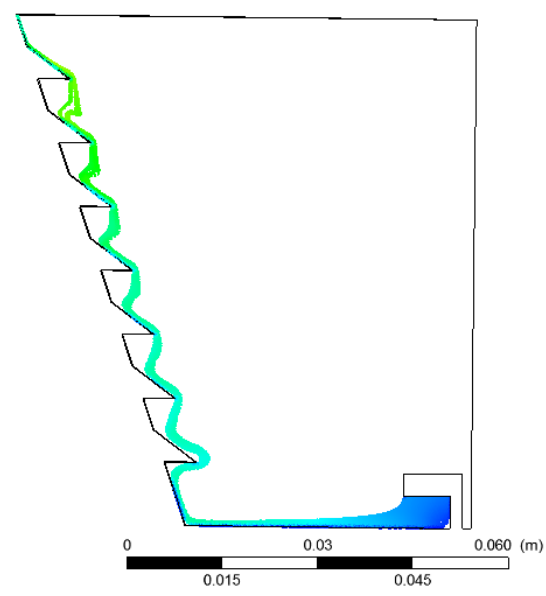

Fig. 6. Flow lines in the concentrator No. 1 


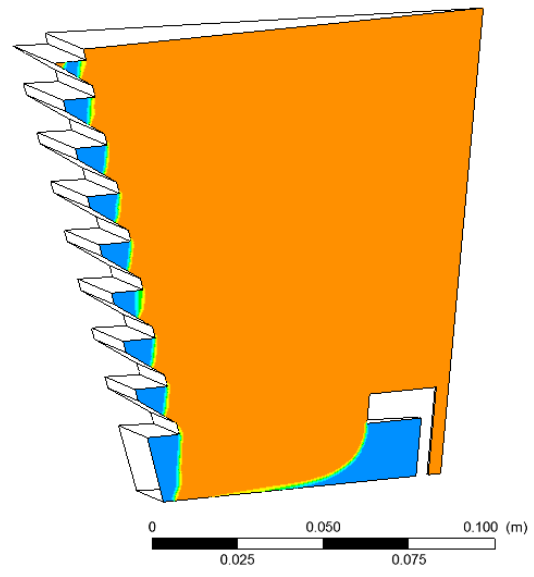

Fig.7. Flow pattern in the concentrator No. 2

\subsection{Verification of the model}

The absence of similar studies of the hydraulic characteristics of centrifugal concentrators presents a problem for comparative evaluation of the applicability of the chosen mathematical model. In our case, verification was carried out based on the problem of determining the form of free liquid surface in a rotating vessel. Preliminary modeling showed that using the developed model allows obtaining flow patterns adequate to the calculated data. The form of free liquid surface in a rotating vessel is shown in Fig. 8. The results of the comparison of numerical experiment data with the approved theoretical calculation method are presented in Fig. 9 and 10.

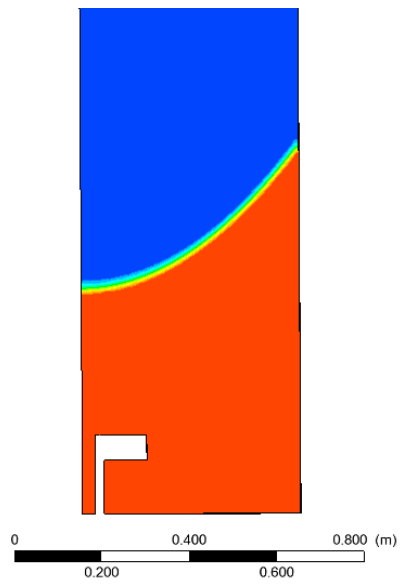

Fig. 8. The form of free liquid surface in a rotating vessel 


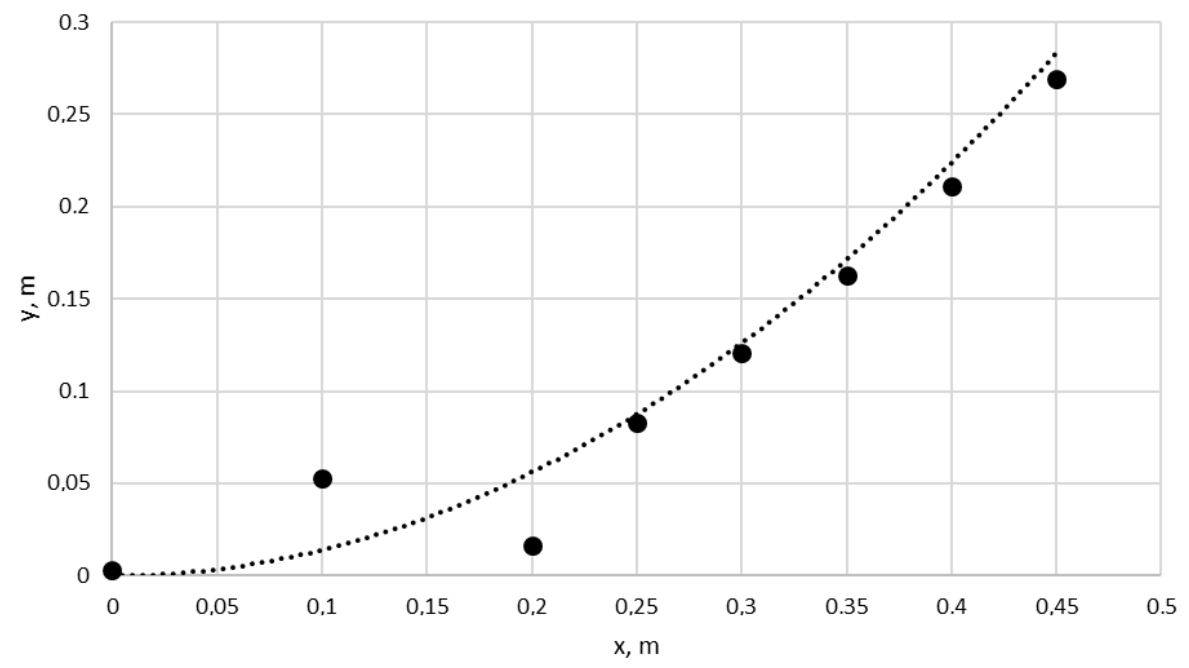

Fig. 9. Comparison of the coordinates of free liquid surface obtained by numerical simulation with calculations data at $50 \mathrm{rpm}$

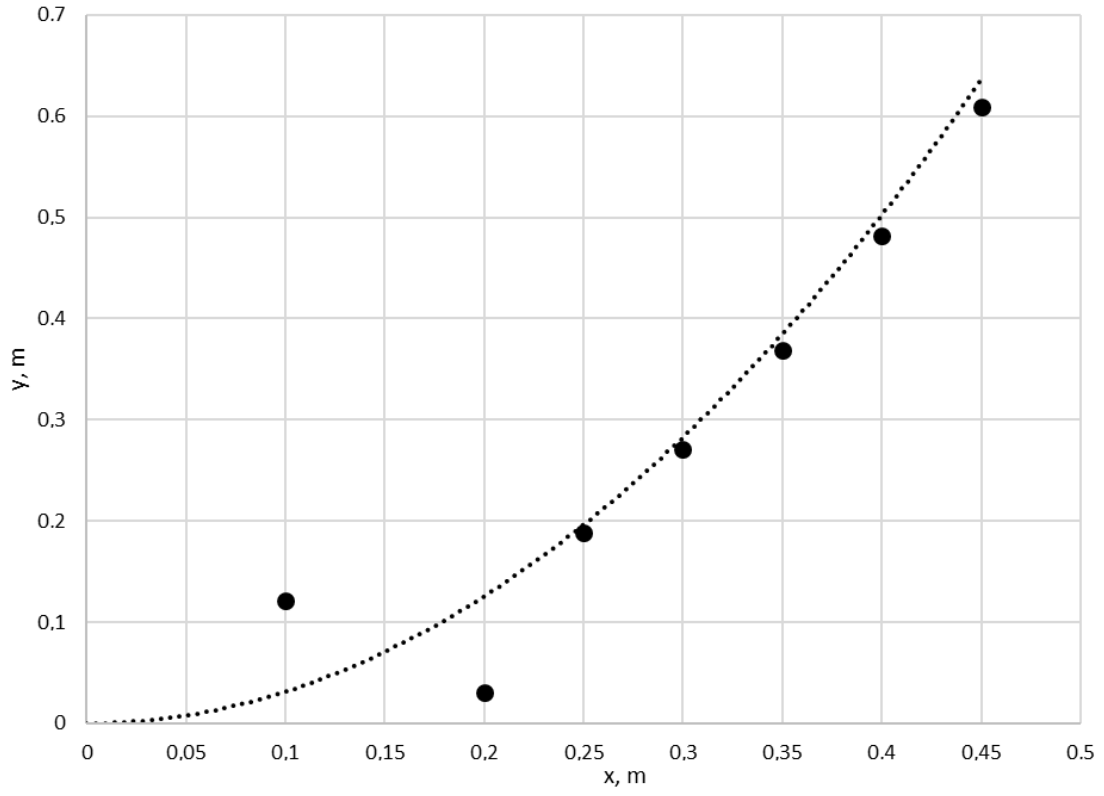

Fig. 10. Comparison of the coordinates of free liquid surface obtained by numerical simulation with calculations data at $75 \mathrm{rpm}$

\section{Conclusion}

As a result of preliminary studies, a mathematical model was chosen for computer simulation of rotating flows with a free surface. The numerical model was successfully tested for the problem of determining free liquid surface in a rotating vessel. Using the numerical method will make it possible to: 
1. Study the work of concentrators of various sizes and designs under different operating conditions, with a change in hydraulic characteristics (speed of treated liquid, speed of rotation of the bowl).

2. In the future study the problems of depositing suspended particles of specified characteristics (density, size) in a centrifugal field and determine the concentration of the sediment in the treatment process.

It should be noted that the current model needs improvement, as it does not allow to study concentrators with vibration, deformation of the bowl surface (floating bed), with complex movement of the concentrator bowl (rotational, not radial) and the use of magnetic field.

At the same time, its application will increase the output of a centrifugal concentrator and determine optimal regimes for the enrichment of ash and slag waste of various chemical compositions.

\section{Acknowledgments}

This paper was prepared with the financial support of the Ministry of Education and Science of the Russian Federation, state task in the field of scientific activity № 10.3706.2017/4.6.

\section{References}

1. Critical of mineral raw materials and sustainability assessment, Expert Workshop on Security of Supply and Scarcity of Raw Materials (2012).

2. A.S. Kobzev Directions of development and problems of radiometric methods of mineral dressing, Enrichment of ores, 1. (2013).

3. V.C. Nguyen, A.V. Petrov Technical and economic simulation of technological mineral dressing processes, Proceedings of the 12th International workshop on Computer Science and Information Technologies, 1. (2010).

4. R. Peter King Modeling \& Simulation of Mineral Processing Systems (2011).

5. L. Ergun, Z. Ekmekci, O. Gulsoy and H. Benzer Modeling and Simulation of the grinding circuit in the Madneuli copper concentrator, Physiochemical Problems of Mineral Processing, 38 (231) (2004).

6. A.Oleynik, V. Skorokhodov Modeling and modeling in the mineral processing industry, Annals of DAAAM for 2006 \& Proceedings of the $17^{\text {th }}$ International DAAAM Symposium, 1 (17). (2006).

7. O.V. Friedman Information technology of the organization of processes of mineral raw materials enrichment, Information resources of Russia, 3. (2006). 